\title{
Identification of edge-localized moments of the current density profile in a tokamak equilibrium from external magnetic measurements.
}

\author{
P.J. Mc Carthy \\ Department of Physics, University College Cork, Association Euratom-DCU, Cork, \\ Ireland \\ E-mail: pjm@ucc.ie
}

\section{ASDEX Upgrade Team}

Max Planck Institut für Plasmaphysik, Boltzmannstrasse 2, D-85748 Garching,

Germany

\begin{abstract}
.
The flux surface topology of a toroidal plasma bounded by a magnetic separatrix allows edge moments of the toroidal current density profile to be identified in an MHD equilibrium reconstruction code using only external magnetic measurements. This is demonstrated analytically for simple plasma shapes and applied to experimental data on the ASDEX Upgrade tokamak where CLISTE reconstructions from magnetic data are shown to be consistent with those obtained from a more complete set of diagnostic data. An independent demonstration of edge current profile recoverability is obtained by analyzing the reconstruction errors for a database of Monte Carlogenerated equilibria.
\end{abstract}

(Some figures in this article are in colour only in the electronic version) 


\section{Introduction}

Equilibrium magnetic measurements (or "magnetics") outside the plasma provide essential information, including the identification of the plasma boundary surface, for MHD equilibrium reconstruction on tokamaks and other magnetically confined plasma experiments. Iterative equilibrium reconstruction from magnetic data was first developed on the Doublet III tokamak [1], where optimization of free parameters to fit the raw data in a least squares sense required a computationally expensive full equilibrium solution at each iteration. The algorithm was speeded up considerably by the interleaving of force balance convergence and a linear regression to determine optimum values of the free parameters at each cycle, resulting in the computationally efficient EFIT equilibrium reconstruction code [2, 3], now the most widely used worldwide. More recently, Bayesian methods have been applied to equilibrium reconstruction from multiple diagnostics on stellarators [4] and tokamaks [5, 6]. Starting with Lackner's fast equilibrium solver based on the Buneman algorithm [7], the CLISTE interpretive equilibrium code $[12,13]$, which is used here, was developed for equilibrium reconstruction from multiple diagnostics on the ASDEX Upgrade tokamak. While it uses the same interleaving algorithm as EFIT, there are significant differences between the two codes which are outlined in section 4 below.

It has been a standard assumption $[2,9,10]$ that information on the internal current density and pressure profiles provided by magnetics is limited to three integral moments, namely the toroidal plasma current $I_{p}=\int_{A} j_{\phi} d A$, beta poloidal $\beta_{p}=2 \mu_{0} \int_{V} p d V /\left(V \bar{B}_{\theta_{b}}^{2}\right)$ and internal inductance $l_{i}=\int_{V} B_{\theta}^{2} d V /\left(V \bar{B}_{\theta_{b}}^{2}\right)$ where $j_{\phi}$ is the toroidal current density, $\bar{B}_{\theta_{b}}=\mu_{0} I_{p} / \int_{b} d \ell$ is the average value of the poloidal magnetic field on the boundary $b$, and $V$ and $A$ denote the plasma volume and cross-sectional area. Here we present analysis and results from reconstructions of a database of randomly generated ASDEX Upgrade equilibria as well as from experimental data which show that additional moments of $j_{\phi}$, strongly localized in the edge region of the plasma, are identifiable from magnetics when the plasma is bounded by a separatrix with one or more X-points. Before proceeding, however, it is first necessary to address a previous claim [11] that magnetics are unable to identify any details of the current distribution, based on the result that the magnetic field at a point $\boldsymbol{r}$ outside the plasma generated by volume currents flowing in the plasma:

$$
\boldsymbol{B}_{p l}(\boldsymbol{r})=\frac{\mu_{0}}{4 \pi} \int_{V} \boldsymbol{j}\left(\boldsymbol{r}^{\prime}\right) \times \frac{\boldsymbol{r}-\boldsymbol{r}^{\prime}}{\left|\boldsymbol{r}-\boldsymbol{r}^{\prime}\right|^{3}} d V
$$

(the integral is taken over the primed source coordinates) is identical to that produced by a surface current density $\boldsymbol{i}=\boldsymbol{n} \times \boldsymbol{B} / \mu_{0}$

$$
\boldsymbol{B}_{p l}(\boldsymbol{r})=\frac{\mu_{0}}{4 \pi} \int_{S} \boldsymbol{i}\left(\boldsymbol{r}^{\prime}\right) \times \frac{\boldsymbol{r}-\boldsymbol{r}^{\prime}}{\left|\boldsymbol{r}-\boldsymbol{r}^{\prime}\right|^{3}} d S
$$

where $S$ is the plasma boundary surface, $\boldsymbol{n}$ is the unit outward normal on $S$ and the equilibrium field $\boldsymbol{B}=\boldsymbol{B}_{p l}+\boldsymbol{B}_{\text {ext }}$ is the sum of plasma $\left(\boldsymbol{B}_{p l}\right)$ and external $\left(\boldsymbol{B}_{\text {ext }}\right)$ contributions. It is claimed in [11] that this identity, valid for arbitrary current 
distributions satisfying $\boldsymbol{B} \cdot \boldsymbol{n}=0$ on $S$, proves that it is impossible to distinguish, on the basis of magnetics alone, between a volume current distribution $\boldsymbol{j}\left(\boldsymbol{r}^{\prime}\right)$ and the equivalent surface distribution $\boldsymbol{i}\left(\boldsymbol{r}^{\prime}\right)$ and hence that no information on the shape of the $\boldsymbol{j}\left(\boldsymbol{r}^{\prime}\right)$ profile can be recovered. We show here by counterexample that this argument conflicts with the MHD equilibrium requirement that any proposed alternate solution must also satisfy the force balance relation $\boldsymbol{j} \times \boldsymbol{B}=\nabla p$ : Consider the infinite aspect ratio limit where the scalar Grad-Shafranov equation (GSE) of force balance simplifies to

$$
-\nabla^{2} \psi=\mu_{0} \frac{d}{d \psi}\left(p(\psi)+\frac{B_{z}^{2}(\psi)}{2 \mu_{0}}\right) \equiv \mu_{0} j_{z}(\psi)
$$

where $\psi$, the poloidal flux per unit length, is independent of the axial coordinate $z$, and $p, B_{z}$, and $j_{z}$, the plasma pressure, axial magnetic field and axial current density profiles are flux functions. The magnetic field is $\boldsymbol{B}=\nabla \psi \times \hat{\boldsymbol{z}}+B_{z} \hat{\boldsymbol{z}}=B_{\theta} \hat{\boldsymbol{\theta}}+B_{z} \hat{\boldsymbol{z}}$ where the unit vectors $\hat{\boldsymbol{\theta}}, \boldsymbol{n}$ and $\hat{\boldsymbol{z}}$ satisfy $\hat{\boldsymbol{\theta}} \times \boldsymbol{n}=\hat{\boldsymbol{z}}$. The equivalent surface current density $\boldsymbol{i}=\boldsymbol{n} \times \boldsymbol{B} / \mu_{0}=\left(B_{z} \hat{\boldsymbol{\theta}}-B_{\theta} \hat{\boldsymbol{z}}\right) / \mu_{0}$ has an axial component $i_{z}=-B_{\theta} / \mu_{0}$. Except for the degenerate case of circular flux surfaces where $B_{\theta}$ is independent of $\theta, i_{z}$ varies with $\theta$ on a surface of constant $\psi$ and hence violates the conditions for force balance, thereby invalidating the reasoning leading to the main conclusion in [11].

\section{Demonstration of edge current identifiability using an analytical model}

The identifiability of edge moments of the $j_{\phi}$ profile can be demonstrated from the following simple analytic model: The flux function per unit length for two parallel wires along the $z$ direction which pass through $x=0, y= \pm d$ and carry equal current $I$ is

$$
\Psi:(x, y)=\frac{\mu_{0} I}{2 \pi} \ln \frac{d^{2}}{\sqrt{x^{2}+(y-d)^{2}} \sqrt{x^{2}+(y+d)^{2}}}
$$

The doublet shape has a single $\mathrm{X}$-point at the origin (where $\Psi=0$ ) and the upper separatrix contour is bounded by $-d / 2 \leq x \leq d / 2$ and $0 \leq y \leq \sqrt{2} d$. Figure 1(a) shows the upper separatrix and an internal contour for the case of $d=1$. The crosssectional area $A(\rho)$ can be evaluated analytically as follows: Working with the scaled flux $\psi \equiv 2 \pi \Psi / \mu_{0} I$, the $x$-coordinate at the position of widest horizontal extent of the contour labelled by $\psi$ is obtained by solving the equation $d x / d y=0$ for fixed $\psi$. This

yields $x_{w}= \pm \sqrt{d^{2}-y\left(x_{w}\right)^{2}}$ where $y\left(x_{w}\right)$ is the corresponding $y$-coordinate. Denoting the positive solution for $x_{w}$ by $r$, the flux surface horizontal radius, and making the substitution $y(r)= \pm \sqrt{d^{2}-r^{2}}$ in eq. (4) we solve for $r$ and obtain

$$
r(\psi)=d e^{-\psi} / 2
$$

which takes the value $r(0)=d / 2$ at the separatrix. Defining the normalized flux radius $\rho=2 r / d=e^{-\psi}$ and setting $x=0$ in eq. (4) we obtain the top and bottom $y$-coordinates for each flux contour:

$$
y_{\text {top }}(\psi)=d \sqrt{1+\rho} ; \quad y_{b o t}(\psi)=d \sqrt{1-\rho}
$$


Using eq. (4) to express $x$ in terms of $y$ and $\psi$ :

$$
x(y, \psi)= \pm \sqrt{2 d \sqrt{r(\psi)^{2}+y^{2}}-d^{2}-y^{2}}
$$

the cross-sectional area integral for the $\psi$ contour

$$
A(\psi)=2 \int_{y_{\text {bot }}}^{y_{t o p}} x_{+}(y, \psi) d y
$$

(where $x_{+}$is the positive root in eq. (7)) may be carried out with the help of the substitution $u=\sqrt{r^{2}+y^{2}}$ when eq. (8) becomes

$$
A(\psi)=2 \int_{d-r}^{d+r} u \sqrt{\frac{r^{2}-(u-d)^{2}}{u^{2}-r^{2}}} d u
$$

This integral was evaluated, with the aid of Mathematica[8], to yield the expression for area (in terms of $\rho$ ):

$$
A(\rho)=d^{2} \sqrt{1-\rho^{2}}\left(E\left[\left(1-\rho^{-2}\right)^{-1}\right]-K\left[\left(1-\rho^{-2}\right)^{-1}\right]\right)
$$

where $K$ and $E$ are complete elliptic integrals of the first and second kind, and $\lim _{\rho \rightarrow 1} A(\rho)=d^{2}$. Evaluation of the flux surface average of the $y$ coordinate as a
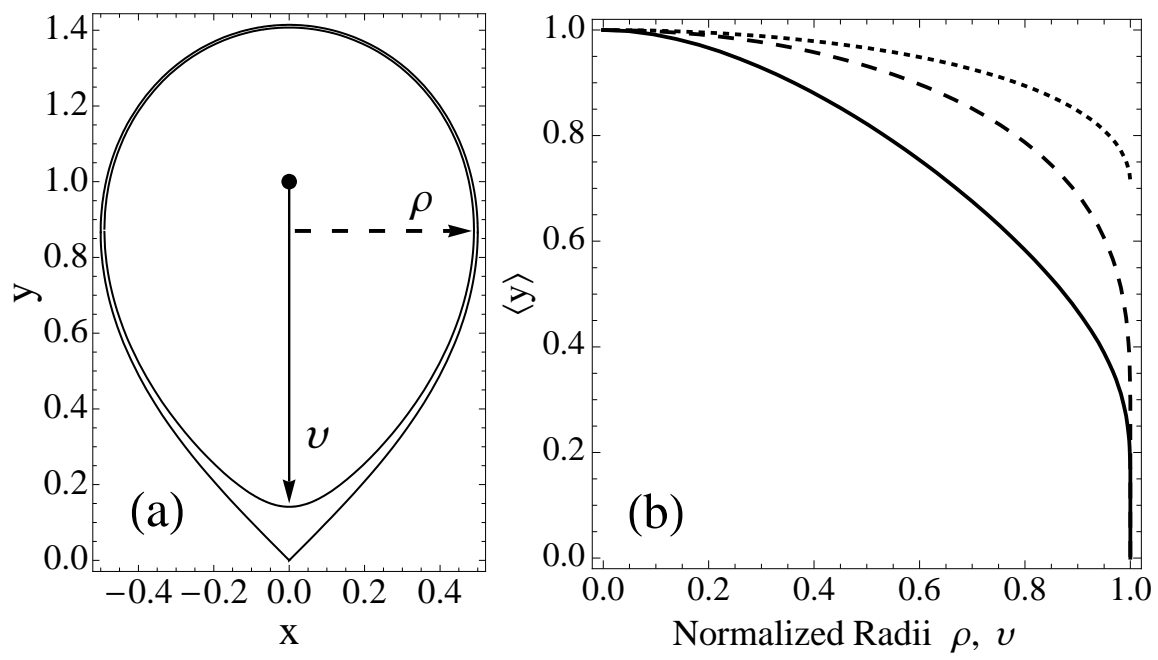

Figure 1. (a) Upper separatrix and $\rho=0.98$ flux contours for wire currents located at $y= \pm 1 \mathrm{~m}$. (b) Geometric mean (...) and flux surface average $\langle y\rangle(---)$ versus $\rho$, the normalized horizontal radius, and $\langle y\rangle$ versus $v(-)$, the normalized distance from the wire to the point of intersection with the flux surface along the line joining the wire to the X-point. Both vertical axes and the left horizontal axis are dimensioned in $m$.

function of $\rho$ yields the exact analytic result

$$
\langle y\rangle_{\rho}=\oint \frac{y d \ell}{|\nabla \psi|} / \oint \frac{d \ell}{|\nabla \psi|}=\frac{\pi d \sqrt{1-\rho^{2}}}{2 K\left[\left(1-\rho^{-2}\right)^{-1}\right]}
$$

The dotted curve in figure 1(b) shows the mild behaviour of the geometric mean of $y$ as a function of $\rho$. In contrast, $\langle y\rangle_{\rho} \sim-1 / \ln (1-\rho)$ as $\rho \rightarrow 1$ and so the dashed curve falls to zero at $\rho=1$ due to the progressive localization around the X-point of the area in 
the annular interval $\{\rho, 1\}$. This localization is seen to extend further into the plasma when $\langle y\rangle$ is plotted (solid curve in figure $1(\mathrm{~b})$ ) versus the radial coordinate $v$ defined as the normalized distance to the point of intersection with the flux surface along the line joining the magnetic axis to the X-point. For the wire model, $v=1-\sqrt{1-\rho}$.

The qualitative features in the above analysis apply to X-point tokamak equilibria whose flux surfaces possess the same essential topological features. In particular, the localization of near-separatrix annular areas to the region just above the X-point (as demonstrated by the behaviour of $\langle y\rangle$ in figure 1(b) ) allows the identification of edge moments of $j_{\phi}$, since the current flowing in this region behaves like a local distribution near the $\mathrm{X}$-point which is distinct from the main current distribution and therefore identifiable by magnetics.

\section{SVD analysis of current profile influence on probe data}

For quantitative results we consider an ASDEX Upgrade single null equilibrium (figure $2(\mathrm{a})$ ) with tangential and normal magnetic probes located on an idealized measurement contour conformal to the separatrix but scaled up by $40 \%$, a factor typical for probeseparatrix distances. At each flux surface we calculate the poloidal field components $B_{R}, B_{Z}$ at each probe site for toroidal current flowing in a thin annulus. If $A^{\prime}=d A / d \psi$ is the area derivative profile with respect to poloidal flux per radian $\psi$, and $d^{2} A / d \psi d \ell_{\theta} \equiv$ $1 /|\nabla \psi|$ is the variation of area with respect to $\psi$ and poloidal arc length $\ell_{\theta}$, then the contribution to the poloidal field components $B_{R}, B_{Z}$ at probe location $\left(R_{p}, Z_{p}\right)$ due to a small annular current $\delta I_{a}$ is given by the poloidal contour integral

$$
\delta B_{R, Z}\left(R_{p}, Z_{p}\right)=\frac{\delta I_{a}}{A^{\prime}} \oint \frac{G_{R, Z}\left(R_{p}, R, Z_{p}, Z\right)}{|\nabla \psi|} d \ell_{\theta}
$$

where the Green's function terms for the field components at the probe location $\left(R_{p}, Z_{p}\right)$ due to unit current at the location $(R, Z)$ are given by[14]

$$
\begin{aligned}
G_{R} & =\frac{\mu_{0}}{4 \pi} \frac{\left(Z_{p}-Z\right)}{\sqrt{R_{p}^{3} R}} \frac{k}{\left(1-k^{2}\right)}\left[\left(1-\frac{k^{2}}{2}\right) E\left(k^{2}\right)-\left(1-k^{2}\right) K\left(k^{2}\right)\right] \\
G_{Z} & =\frac{\mu_{0}}{8 \pi} \sqrt{\frac{R}{R_{p}^{3}}} \frac{k}{\left(1-k^{2}\right)}\left[\left(k^{2}-\frac{2 R_{p}}{R}\left(1-\frac{k^{2}}{2}\right)\right) E\left(k^{2}\right)+\frac{2 R_{p}}{R}\left(1-k^{2}\right) K\left(k^{2}\right)\right]
\end{aligned}
$$

and the argument $k^{2}$ is given by

$$
k^{2}=\frac{4 R_{p} R}{\left(R_{p}+R\right)^{2}+\left(Z_{p}-Z\right)^{2}}
$$

Note that a constant current density in the annulus, equivalent to the flux surface averaged value $\left\langle j_{\phi}\right\rangle$, has been assumed in this section in the interest of simplicity. Flux surface contour integrals of the Greens function versus $v$ at each of $P$ probe sites were numerically evaluated to generate $2 P$ radial influence profiles. 

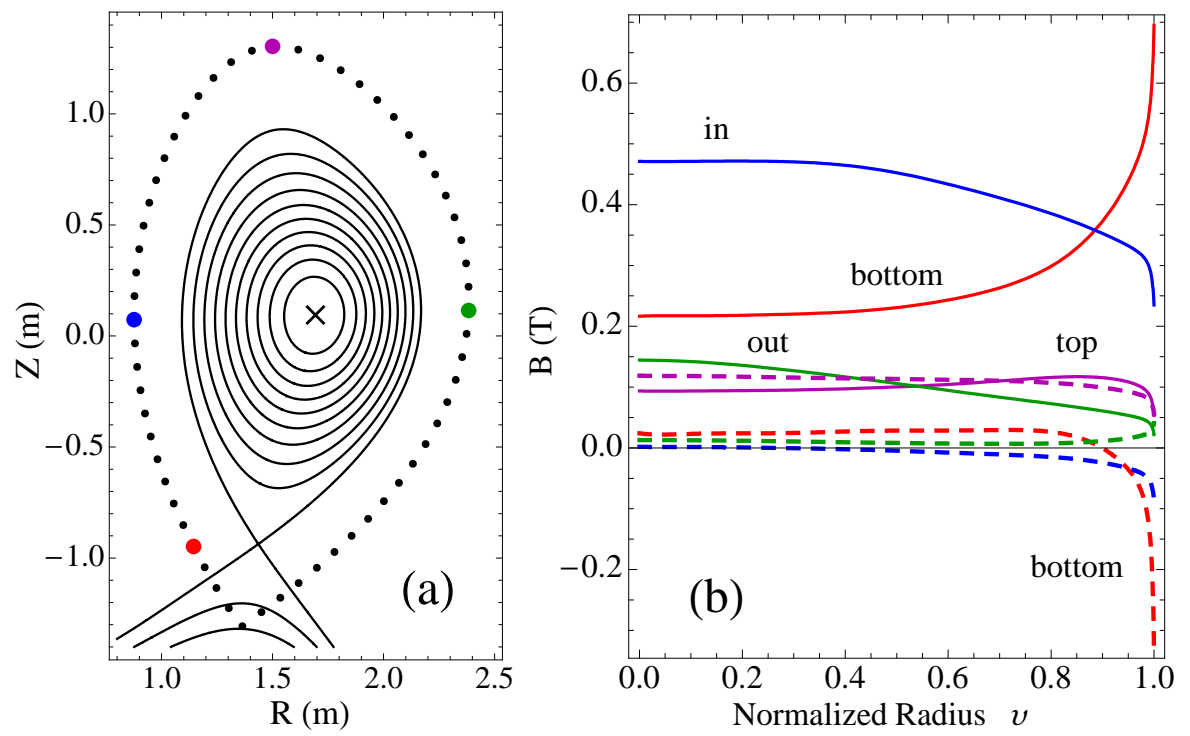

Figure 2. (colour online) (a) Lower null ASDEX Upgrade equilibrium with $P=60$ equidistant magnetic probe sites on an idealized measurement contour conformal with the separatrix but scaled up by $40 \%$. (b) tangential (-) and normal (- - ) components of the poloidal magnetic field per MA annular current for the four sites highlighted in figure 2(a) versus the flux label $v$ (see figure 1 caption).

Sample profiles plotted in figure 2(b) all have the property that they are nearly independent of $v$ in the plasma core, but change, very dramatically in the case of probes near the $\mathrm{X}$-point, as $v \rightarrow 1$. It is this strong variation towards the plasma boundary (a consequence of the behaviour of $\langle y\rangle$ near the separatrix in figure 1(b))) that enables identification of edge moments of $j_{\phi}$. The influence profiles were discretized into $N$ radial elements to form an $N \times 2 P$ influence matrix $\mathcal{G}$. The Singular Value Decomposition $(\mathrm{SVD})$ of $\mathcal{G}$ provides a sequence of left-singular vectors $\mathbf{u}_{i}$ representing orthogonal moments of the $\left\langle j_{\phi}\right\rangle(v)$ profile, a sequence of right-singular vectors $\mathbf{v}_{i}$ of corresponding orthogonal moments of the probe measurements and a set of singular values $\sigma_{i}$, in tesla, whose magnitudes, when compared to experimentally known measurement uncertainty, give a practical measure of the recoverability of the corresponding moments of the current profile.

Figure 3 plots the radial dependence of the leading six left-singular vectors, labelled by their singular values, which were generated by the SVD of an $N \times 2 P=400 \times 120$ matrix where the influence of each annulus on the contour of probe measurements was scaled by the annular area (this was necessary because the choice of equidistant intervals in $v$ resulted in variable area annuli) and the plasma current was 1 MA. Corresponding right-hand singular vectors are represented by spatial distributions of dot sizes in the right panel of figure 3. Left-singular vectors 1 and 2, which correspond to $I_{p}$ and $l_{i}$, depend on the entire current distribution. Subsequent singular vectors are predominantly sensitive to the edge region $v \gtrsim 0.9$ or $\rho \gtrsim 0.99$. The singular values satisfy $\Sigma_{i} \sigma_{i}^{2}=\Sigma_{j=1}^{P}\left(B_{j, \|}^{2}+B_{j, \perp}^{2}\right)$ where $B_{j, \|}$ and $B_{j, \perp}$ are the components of the 

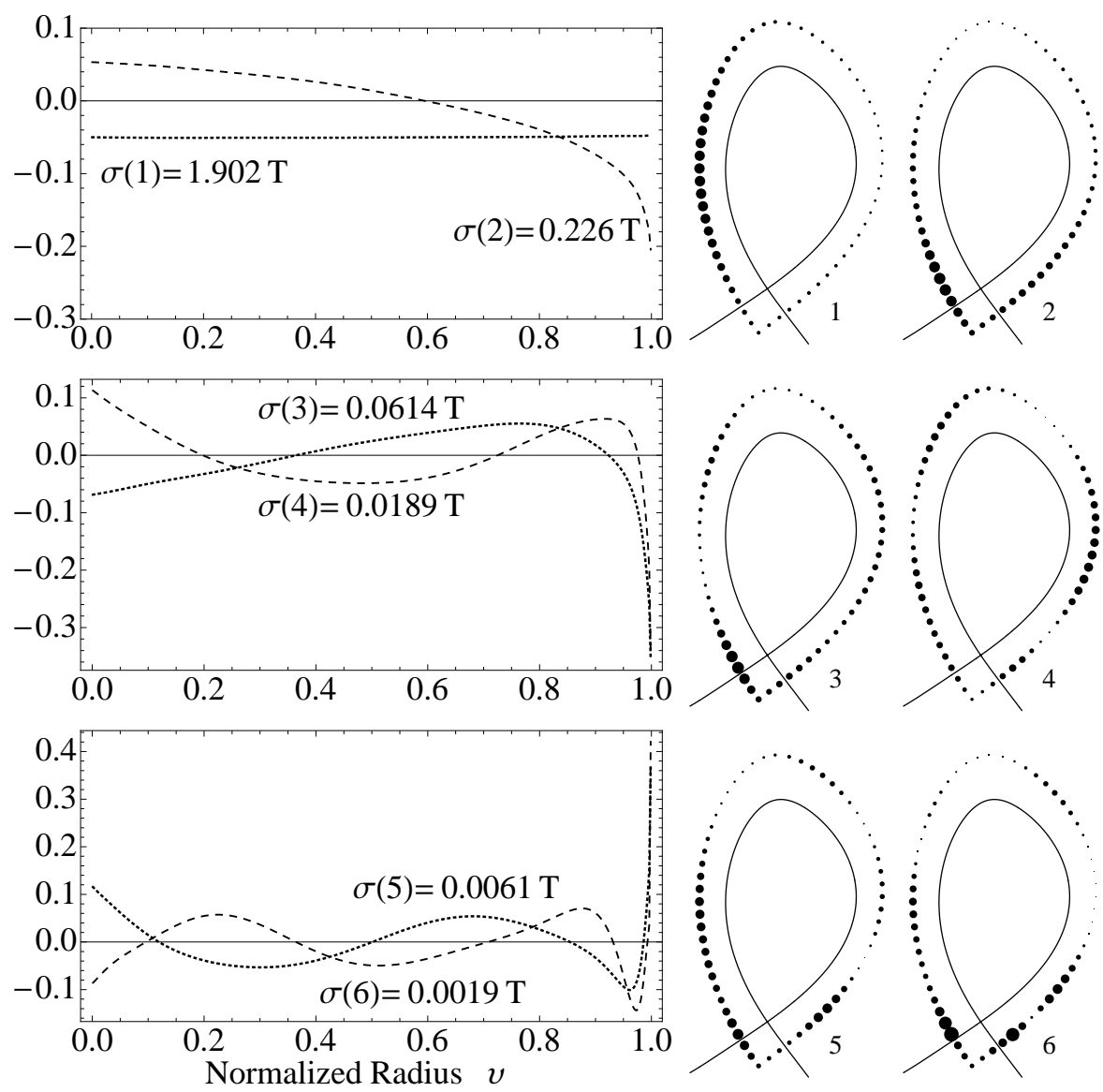

Figure 3. Left panel: Leading six left-singular vectors and singular values from an SVD of the matrix of radial influence profiles. Right panel: Right-singular vectors, the magnitude of whose elements are represented by dot size at each probe site: For the $i^{\text {th }}$ right-singular vector $\mathbf{v}_{i}$, the dot area at probe site $j$ is $\propto \mathbf{v}_{i, j}^{2}+\mathbf{v}_{i, j+P}^{2}$, i.e. the sum of squared weights for the two poloidal field components at site $j$.

poloidal magnetic field at the $j$ th probe site due to a 1 MA plasma current. For variable plasma current $I_{p}$, ratio of probe contour circumference to separatrix circumference $r$, and number of probe sites $P$, the second and subsequent singular values were observed to scale approximately as $\sigma_{j} \sim \sqrt{P} I_{p} /(2 r)^{j}$. The orthogonality of the singular vectors and their ordering in terms of experimental recoverability make them attractive in principle as radial basis functions in the MHD equilibrium identification problem.

\section{Edge current identifiability using the CLISTE equilibrium code}

The foregoing analysis is incomplete in several respects: A fixed flux surface topology is used and hence the known dependence of magnetics on the Shafranov shift is not reflected in the singular vectors. Also, the use of $\left\langle j_{\phi}\right\rangle$ conceals the major radius dependence of the $j_{\phi}$ profile. Current flow in the scrape-off layer (SOL) outside the separatrix is not considered. These aspects are all taken into account in the 
CLISTE interpretive equilibrium code $[12,13,15]$ which generates MHD equilibrium solutions on ASDEX Upgrade constrained by data from multiple diagnostics. CLISTE was developed from the predictive Garching Equilibrium Code, originally written by Lackner, which includes a fast equilibrium solver based on the Buneman algorithm [7]. The fast interleaving algorithm of EFIT is also implemented in CLISTE, however there are differences between the two codes: EFIT uses pre-calculated Green's function matrices containing the response at each measurement site to unit plasma current at each gridpoint, whereas CLISTE solves the Poisson problem for the poloidal flux generated by each current profile basis function at each iteration cycle. EFIT uses a tension spline model[20] to parameterize the $p^{\prime}(\psi)$ and $f f^{\prime}(\psi)$ source profiles whereas CLISTE uses cubic splines with curvature regularization. Unlike EFIT, source profiles routinely extend to open field lines in the SOL where they can be constrained using pressure data and shunt resistance measurements of poloidal currents flowing through tiles in the axisymmetric divertor[21]. Constant pressure and force balance is assumed to hold along open fieldlines until a vessel structure is encountered whereupon the plasma current density and pressure fall to zero across one grid interval. The return current through the material structure is assumed to have negligible effect on force balance in the plasma.

The SVD analysis of the previous section demonstrates that magnetics contain information on the edge current distribution, however use of the present SVD approach under realistic conditions for solving the equilibrium problem proved impractical and instead, for modelling flexibility, a cubic spline model with, typically, 10 radial knots is used to parameterize the $p^{\prime}(\psi)$ and $f f^{\prime}(\psi)$ source profile shapes. This amounts to 20 free shape parameters, well in excess of the number of identifiable moments of the current distribution but with the advantage of being free of the bias that commonly affects parameterizations with a low number of free parameters: Overly restricted models can result in artificially well determined pressure and current density profiles using only magnetic data, as was correctly pointed out in [11]. The choice of 10 knot locations has the dual purpose of (a) ensuring that, apart from the effects of regularization, the model is free of significant bias and (b) allowing the same model to be used when the equilibrium solution is additionally constrained by kinetic profile data or other internal diagnostic data where many knots may be required to satisfactorily model the data. Further increasing the number of knots typically does not improve the goodness of fit, and can cause convergence problems. Regularization in the form of curvature penalties at each knot location and, optionally, penalizing the magnitude of the fitted spline coefficients controls the ill-conditioned nature of the problem, the most serious cause of which is the low distinguishability of $p^{\prime}(\psi)$ and $f f^{\prime}(\psi)$ when the solution is constrained only by magnetics. The magnitude of the regularization can be selected to satisfy Morozov's discrepancy principle [16], where the average fit error is not allowed to fall below the experimentally known uncertainty level in the magnetic data. Alternatively, regularization parameter selection methods which do not require prior knowledge of diagnostic error levels such as the L-curve [17] may also be used. 
The recoverability of edge moments of the current density profile is illustrated by a sequence of CLISTE equilibria for ASDEX Upgrade discharge 23255, $I_{p}=800 \mathrm{kA}$, $B_{t}=-2.5 \mathrm{~T}, \bar{n}_{e}=5.8 \times 10^{19} \mathrm{~m}^{-3}$ which had a stationary low power phase consisting of 0.3 MW ohmic heating and 0.5 MW ECRH, followed by the addition of four 2.5 MW neutral beams at $200 \mathrm{~ms}$ intervals (see figure 4) which yielded a factor of six variation in the stored energy during the current flattop phase. A spline model with 10 knots

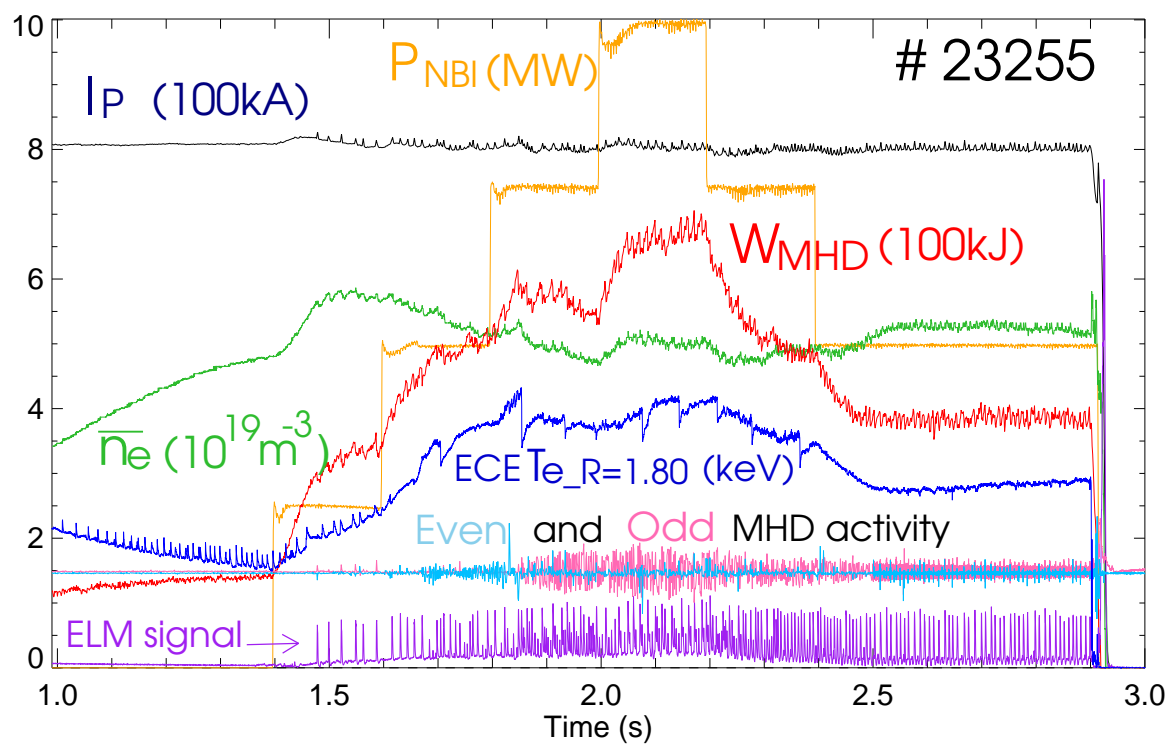

Figure 4. (colour online) Time traces of plasma current $I_{\mathrm{p}}$, line-integrated density $\bar{n}_{e}$, neutral beam injection heating power $P_{\mathrm{NBI}}$, plasma stored energy $W_{\mathrm{MHD}}$, electron temperature $T_{e}$ at major radius $R=1.8 \mathrm{~m}$, MHD even and odd mode signals, and divertor tile currents (ELM signal) for ASDEX Upgrade discharge \# 23255. The CLISTE analysis is for the time window $1.2 \leq t \leq 2.7 \mathrm{~s}$.

for the $p^{\prime}(\psi)$ and $f f^{\prime}(\psi)$ source profiles regularized by penalizing both the curvature at each knot location and also the magnitude of the fitted coefficients was used to find free boundary equilibria constrained by 60 magnetic signals at $10 \mathrm{~ms}$ intervals for the time window $1.2 \leq t \leq 2.7 \mathrm{~s}$, with ELM timepoints excluded. Since sawteeth were present throughout most of the time window, the safety factor $q$ on the magnetic axis was clamped at a value just below unity. The rms (root mean square) fit error was $1.0 \mathrm{mT}$ or $0.9 \%$ of the rms signal magnitude, which is typical of a well-fitted CLISTE reconstruction for $I_{p}=800 \mathrm{kA}$. The same spline model, with identical regularization penalties, was also used to generate equilibria additionally constrained by pressure data in the range $0.85 \leq \rho_{\text {pol }} \leq 1.02$ obtained from smoothed fits to high resolution $n_{e}, T_{e}$ and $T_{i}$ data from the Lithium beam [22] [23], ECE [24], and Thomson scattering [25] diagnostics. The magnetic fit error averaged over the entire time window was unchanged, and the rms pressure fit error was $\approx 150 \mathrm{~Pa}$ or $2.5 \%$ of the rms value of $\approx 6 \mathrm{kPa}$ for $\rho_{\text {pol }} \geq 0.85$.

Figure 5 shows magnetics-only $(\mathrm{M}-\mathrm{O})$ and magnetics+kinetics $(\mathrm{M}+\mathrm{K})$ reconstruc- 

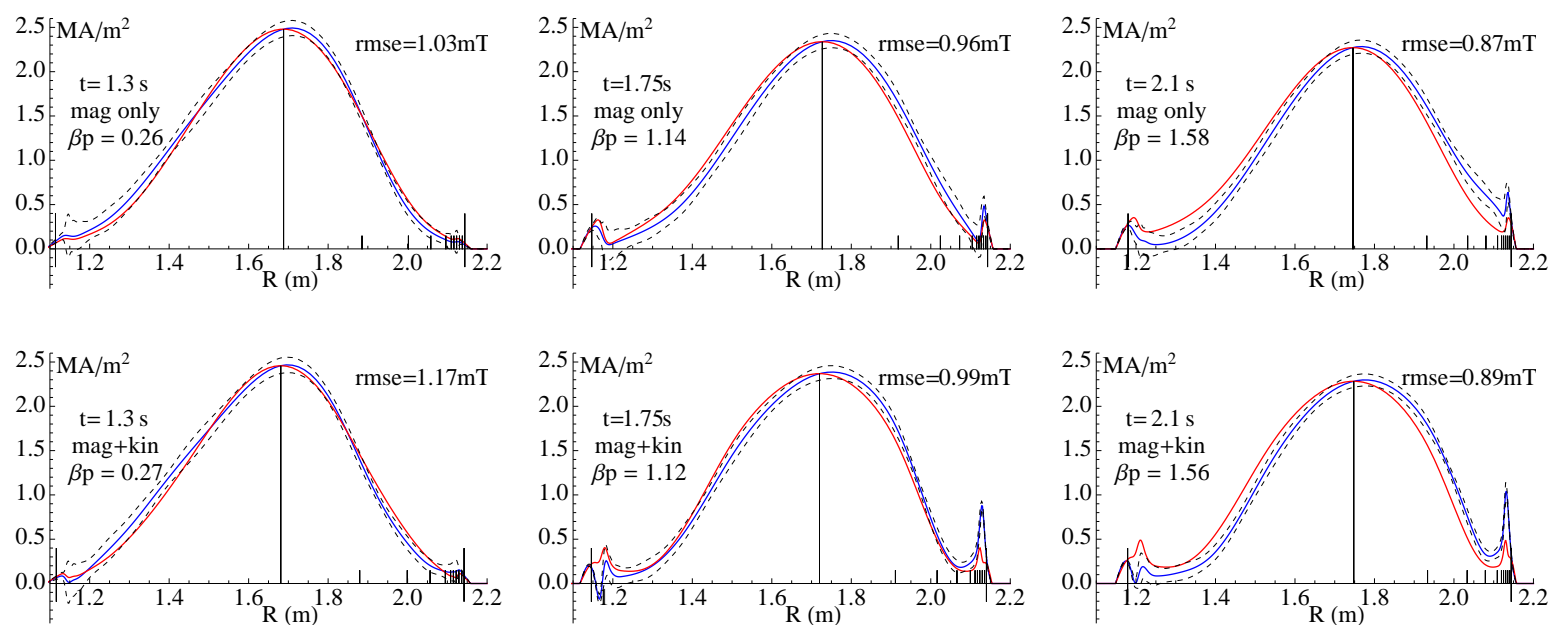

Figure 5. (colour online) Low, medium and high $\beta_{\text {pol }}$ timeslices of $j_{\phi}$ (blue) and $\left\langle j_{\phi}\right\rangle$ (red) profiles as a function of major radius along the magnetic midplane as reconstructed by CLISTE from magnetic data (top row) and a combination of magnetic and edge pressure data (bottom row) for ASDEX Upgrade discharge \# 23255. The magnetic axis position and the inboard and outboard points of intersection with the separatrix are marked by vertical lines. The small vertical markers just above the horizontal axis on the outboard side indicate the positions of the nine internal knots, which are concentrated in the pedestal region where the pressure data is located. The tenth and final end knot coincides with the axis position. The black, dashed profiles are $\pm 1 \sigma$ confidence bands for $j_{\phi}$, see text for further details.

tions of $j_{\phi}$ and $\left\langle j_{\phi}\right\rangle$ profiles for three timeslices, corresponding to low, medium and high $\beta_{\text {pol }}$ values. The black, dashed traces are $\pm 1 \sigma$ confidence bands for $j_{\phi}$ calculated using the variance-covariance matrix $\boldsymbol{V}$ of the linear regression which determines the set of free parameters at the cycle where the convergence criterion is met; see [12] section 5.1 for details. It is important to note that the magnitude of the confidence bands is strongly affected by regularization, which introduces some bias into the solution in return for (strongly) reducing the typically large variance of the unregularized, ill-conditioned problem[17]. Thus care must be taken in interpreting the absolute magnitude of the confidence bands. In contrast, it is more straightforward to compare of the relative magnitudes of the $\mathrm{M}-\mathrm{O}$ and $\mathrm{M}+\mathrm{K}$ confidence bands in figure (5). There is little to distinguish the two cases for the first, low power timeslice. For the $\beta_{\text {pol }}=1.1$ and $\beta_{\text {pol }}=1.6$ timeslices, the $\mathrm{M}+\mathrm{K}$ confidence bands are much narrower than those of the $\mathrm{M}-\mathrm{O}$ fit in the outboard region $2.05<R<2.15$, reflecting the influence of the edge pressure data in constraining the solution. Note that there is only a weak narrowing of the confidence bands in the inboard edge region $1.2<R<1.3$. This is due to a factor of $3-4$ increase in the strength of the force-free current term $f f^{\prime}(\psi) / \mu_{0} R$ relative to the pressure-driven term $R p^{\prime}(\psi)$ in moving from $R \approx 2.1$ to $R \approx 1.2$ combined with the fact that the inclusion of the pressure data has no effect in constraining the free parameters describing the $f f^{\prime}(\psi)$ profile. Note that in all six plots the uncertainty in 
$j_{\phi}$ is very small in the SOL. This is a consequence of the strong constraint provided by divertor tile current data, combined with the absence, for these runs, of any additional degree of freedom in the SOL since all knots are located inside the separatrix.
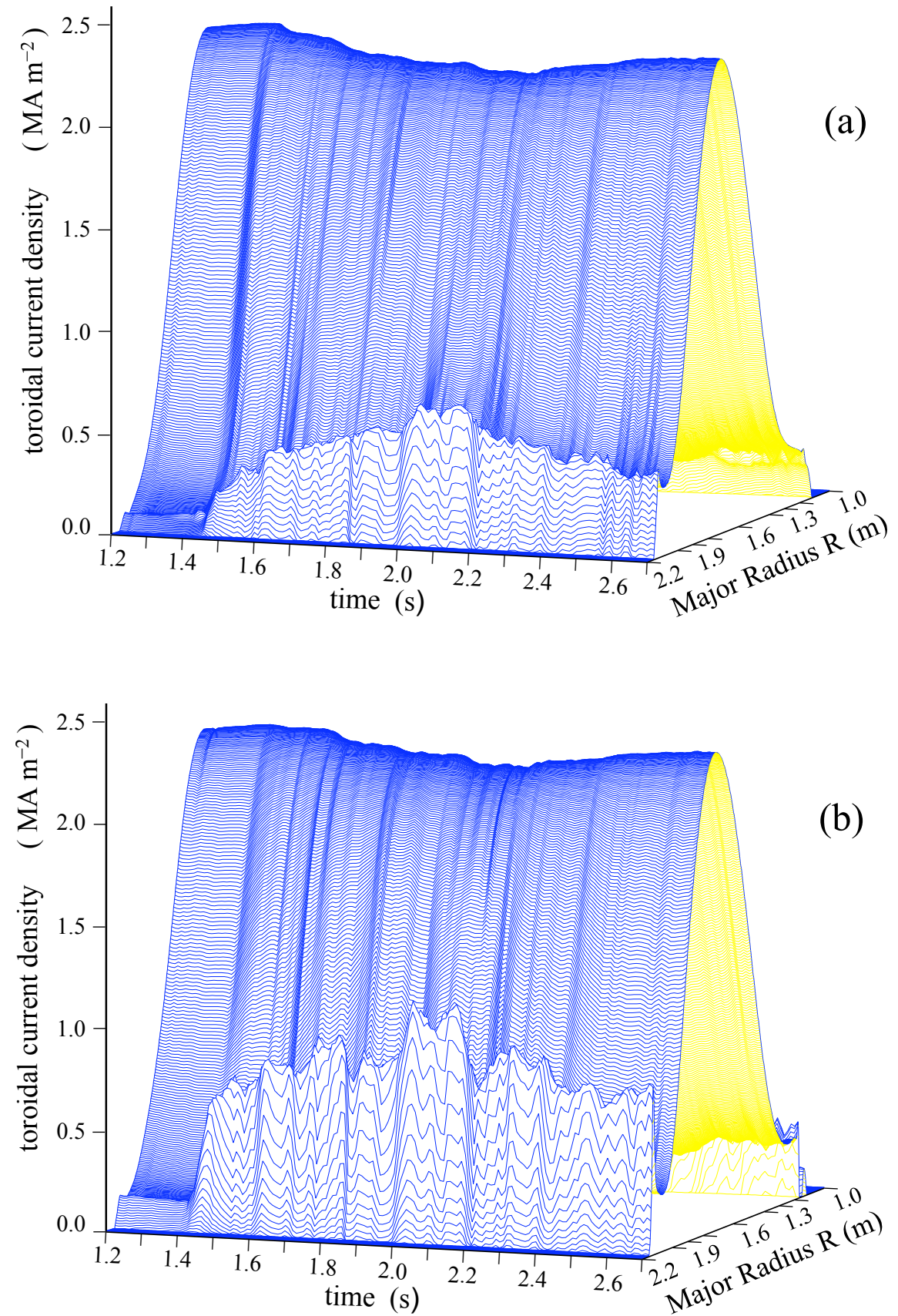

Figure 6. (colour online) Time evolution of the $j_{\phi}$ profile as a function of major radius along the magnetic midplane as reconstructed by CLISTE from (a) magnetic data and (b) a combination of magnetic and edge pressure data.

The full set of reconstructed profiles from 151 individual CLISTE runs for \# 23255 
is presented in figure 6 , which shows the time evolution of the $j_{\phi}$ profile as a function of major radius along the magnetic midplane from CLISTE reconstructions using (a) magnetic data and (b) a combination of magnetic and edge pressure data. In each case, a prominent edge peak in $j_{\phi}$ develops following the start of NBI heating at $t=1.4 \mathrm{~s}$. It increases as further sources are added at $1.6 \mathrm{~s}, 1.8 \mathrm{~s}$ and $2.0 \mathrm{~s}$, reaching a maximum at $t=2.2 \mathrm{~s}$ just as the NBI power is reduced from its peak value. The evolution of the edge peak is very similar in both cases, although the inclusion of the pressure constraints results in the peak height in figure $6(\mathrm{~b})$ exceeding that in $6(\mathrm{a})$ by a factor of $\approx 2$.

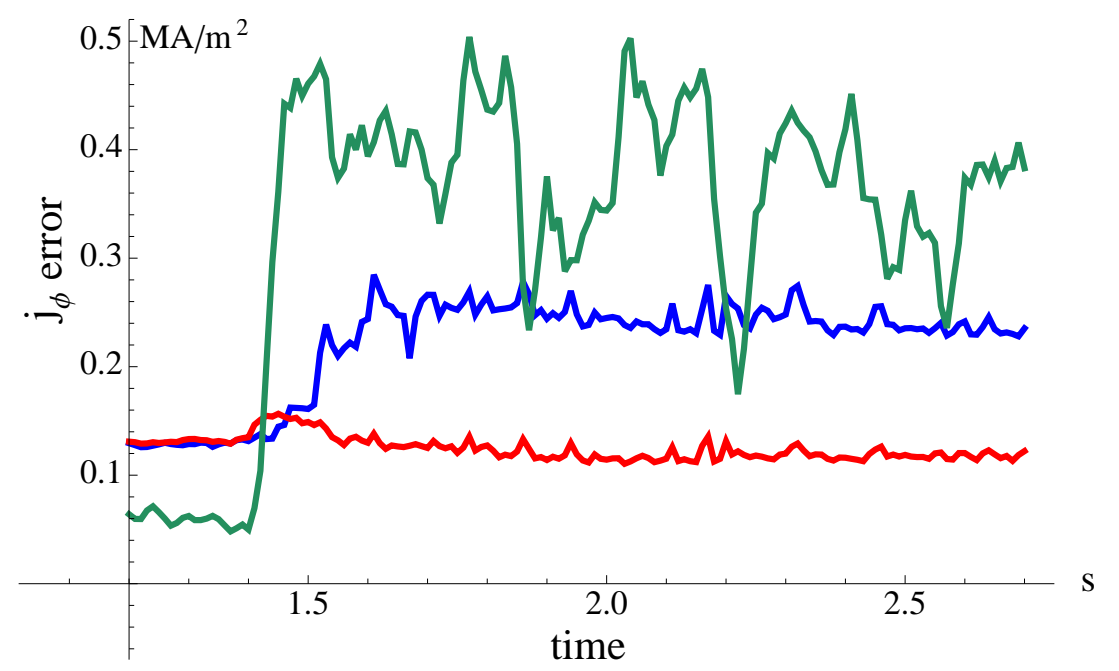

Figure 7. (colour online) Time evolution of the CLISTE $1 \sigma$ confidence band for the outboard peak value of $j_{\phi}$ for the magnetics-only fit (middle, blue trace), and the magnetics+kinetics fit (lower, red trace). Also plotted is the difference between the peak values of the two fits (upper, green trace).

The $1 \sigma$ error bar for the outboard peak $j_{\phi}$ value for both fits is plotted versus time in figure 7 which also shows the difference between the peak values. Under standard statistical assumptions, the $95 \%$ confidence interval for the difference in peak values is the sum of the individual $1 \sigma$ error bars. For $t>1.6 \mathrm{~s}$ this sum is $\simeq 0.40 \mathrm{MA} \mathrm{m}^{-2}$ which is close to the average value of the upper trace in figure 7 . Thus the value of the peak difference is typically marginal at the $95 \%$ confidence level, suggesting that the CLISTE confidence bands may be slightly too restrictive.

Quantitative agreement between both fits is achieved when the toroidal current ' $I_{\text {edge }}$ ' flowing outside a fixed flux surface close to the peak position is calculated in both cases. Figure 8 shows that the current flowing outside the $v=0.9$ flux surface in both cases is nearly identical and scales closely with $\beta_{\text {pol }}$, consistent with a bootstrapdominated current drive in the pedestal. The $1 \sigma I_{\text {edge }}$ error bars of $\pm 2.8 \mathrm{kA}$ and $\pm 2.5 \mathrm{kA}$ for the $\mathrm{M}-\mathrm{O}$ and $\mathrm{M}+\mathrm{K}$ fits were calculated in CLISTE from the final linear regression determining the converged values of the free parameters and were to a good approximation time-independent. 


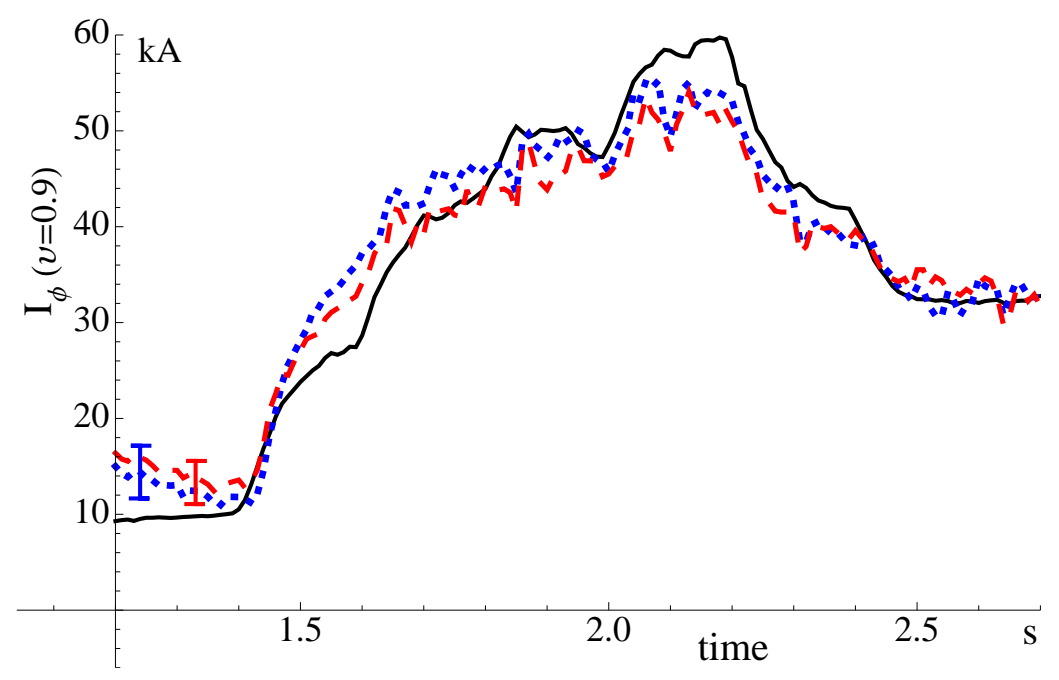

Figure 8. (colour online) Time evolution of toroidal current outside the $v=0.9$ surface (corresponding to $\rho_{\text {pol }} \approx 0.99$ ) for the $\mathrm{M}-\mathrm{O}$ fit (dots) and the magnetics + pressure fit (dashes) with corresponding $1 \sigma$ error bars from CLISTE. The solid curve is $\beta_{p o l}(t)$ scaled by a factor of 37.0 .

\section{Reconstruction of edge current from an equilibrium database}

An independent method of testing the recoverability of edge current profile information is possible at ASDEX Upgrade thanks to the availability of databases of randomly generated equilibria which can be exploited as synthetic experiments with perfect a priori knowledge of the equilibria to be reconstructed by CLISTE. For more than two decades, such databases have been central to the preparation of Function Parameterization (FP) models for rapid identification of equilibrium parameters as part of the ASDEX Upgrade real time feedback control system[14, 15, 18, 19]. Equilibrium databases are generated using a well-developed methodology for populating a highdimensional $(>20 D)$ parameter space with a relatively modest number of cases, typically in the range $10^{3}-10^{4}$. Use of the Monte Carlo approach (where each input parameter value is a random number chosen from a range appropriate to the engineering or physics limits of the input parameter) ensures that the projection onto any 1-D subspace will tend to be smoothly distributed, without large gaps, along that direction in parameter space, thus avoiding the "curse of dimensionality" where the choice of regularly spaced parameter values along each axis would require geometric growth in the number of cases with increasing dimension. Accordingly, for a database of $N$ cases, the Monte Carlo approach has the desirable property that each input parameter and output parameter will take $N$ distinct values in the database.

Using the vacuum vessel and poloidal field coil geometry of ASDEX Upgrade, randomly chosen candidate input parameter vectors specify the currents in the poloidal field coils, the in-vessel passive conductor currents, and the Grad-Shafranov source profiles $p^{\prime}(\psi)$ and $f f^{\prime}(\psi)$. To ensure that only identifiable moments of the current 
profile are recovered from magnetics or other diagnostic data, a strategy of using richly featured source profile shapes with a large number of free parameters has been developed. The $p^{\prime}(\psi)$ and $f f^{\prime}(\psi)$ profiles are parameterized as linear combinations of up to 25 basis functions generated from the Discrete Cosine Transform on a grid consisting of equidistant normalized $\psi$ values in the interval $[0,1]$ where 0 and 1 correspond to the last current-carrying surface (which lies, in general, outside the plasma boundary) and the magnetic axis, respectively. Note that this parameterization of $p^{\prime}(\psi)$ and $f f^{\prime}(\psi)$ is unrelated to the cubic spline model used by CLISTE. The $i$ th component of the $j$ th orthonormal basis function $\mathbf{b}_{\mathbf{j}}$ is given by

$$
b_{i, j}=\sqrt{\frac{\min (j, 2)}{L}} \cos \left((2 i-1)(j-1) \frac{\pi}{2 L}\right) ; \quad i=1, L
$$

where $L=1001$ is the number of $\psi$ gridpoints for the source profiles.

Smoothness conditions are imposed on the selected source functions by use of a spectral decay scheme whereby the amplitude of each profile moment is chosen from a normal probability distribution with zero mean whose standard deviation decreases geometrically with order, so that the finest details of the profile tend to have the lowest amplitude. To ensure that $p^{\prime}(\psi)$ and $f f^{\prime}(\psi)$ tend towards zero in the edge region, the profiles generated using eq. (11) are scaled by a monotonic envelope function $e(\psi)$ (with randomly chosen parameters) which vanishes at the last current-carrying surface. The source profiles are accordingly parameterized by $\sigma(\psi)=e(\psi) \sum_{j=1}^{N_{b}} \alpha_{j} \mathbf{b}_{j}$, where $\sigma(\psi)$ stands for $p^{\prime}(\psi)$ or $f f^{\prime}(\psi), N_{b} \simeq 25$ is the number of linear combinations and $\alpha_{j}$ is the amplitude of the $j$ th Fourier moment. Typically, only a small fraction (several percent) of the candidate input vectors result in acceptable equilibrium solutions. This is due to the fact that most random input parameter combinations will not result in reasonable, or any solutions. The set of acceptable equilibria are stored on disk and constitute the equilibrium database.

A database of 600 cases of $1 \mathrm{MA}$ lower single null equilibria selected to have an almost fixed plasma boundary geometry $\left(\approx 2 \mathrm{~cm}\right.$ variation) but a large variety of $j_{\phi}$ profile shapes and $\beta_{\text {pol }}$ values was used. The database has no SOL currents and $j_{\phi} \rightarrow 0$ at the separatrix. For each equilibrium, the magnetic sensor signals corresponding to the experimentally available set of probes, flux loops and saddle loops was calculated and simulated signal noise was added. These synthetic data were passed to CLISTE, and a reconstruction was made with a 12-knot spline model with knot locations, expressed in terms of the coordinate $\rho_{a}$ (defined below), at the following database averaged values: $(0,0.3,0.45,0.6,0.7,0.77,0.83,0.875,0.91,0.945,0.965,0.98)$. The toroidal current and current density profiles for each reconstructed equilibrium were compared to their exact analogues in the database. Three reconstructions for each equilibrium were calculated using signal noise levels of $\sigma=1 \mathrm{mT}, \sigma=2 \mathrm{mT}$ and $\sigma=3 \mathrm{mT}$. Given the large number of cases, it was possible to construct statistics on the reconstruction of the edge current profile which provide an independent test of the reconstruction accuracy. The flux label $v$ was not available in the database and an alternative geometric label $\rho_{a}$, the radius corresponding to the normalized flux surface cross-sectional area, was chosen as 

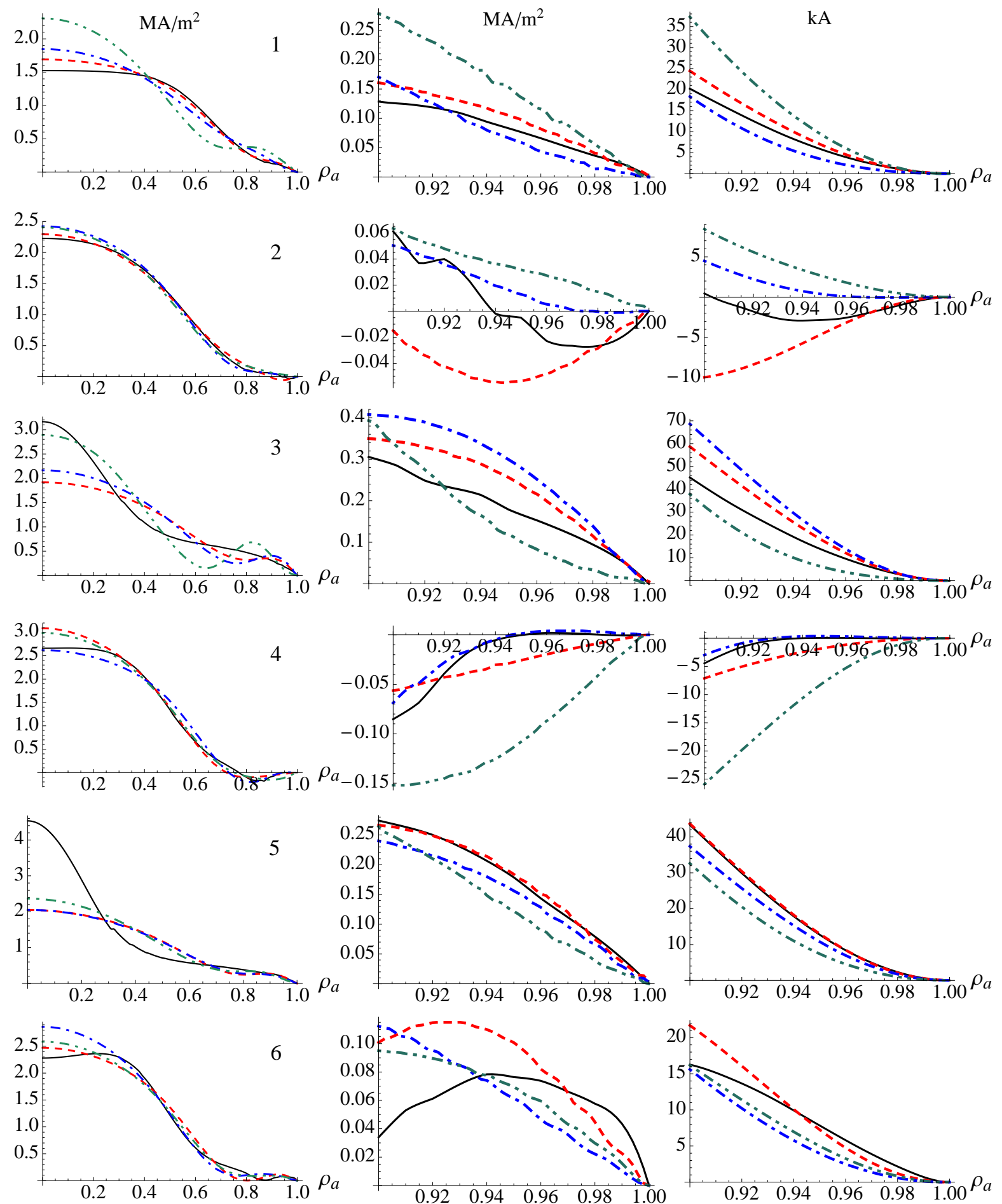

Figure 9. (colour online) Six representative cases from the equilibrium database of exact (solid black traces) and reconstructed flux surface averaged current density $\left\langle j_{\phi}\right\rangle$ and toroidal current $I_{\phi}$ profiles for $1 \sigma$ noise levels of $1 \mathrm{mT}$ (red, dashes ), $2 \mathrm{mT}$ (blue, dash-dot ), and $3 \mathrm{mT}$ (green, dash-dot-dot ). The left-hand column shows the full $\left\langle j_{\phi}\right\rangle$ profile, the middle and right-hand columns zoom in to the edge region $\rho_{a} \geq 0.9$ for $\left\langle j_{\phi}\right\rangle$ and $I_{\phi}$ profiles, respectively. 
the radial coordinate to represent the results. Like other geometry-based flux labels, it has the advantage of being largely independent of the current profile, unlike $\rho_{p o l}$. The database averaged relationship between $\rho_{a}$ and the poloidal flux radius $\rho_{p o l}$ in the edge region is characterised by the following approximate corresponding values for $\left(\rho_{a}, \rho_{\text {pol }}\right):(0.91,0.97),(0.935,0.98),(0.965,0.99),(1,1)$. Profiles for the flux surface averaged current density $\left\langle j_{\phi}\right\rangle\left(\rho_{a}\right)$ and the toroidal current profile $I_{\phi}\left(\rho_{a}\right)$ from CLISTE reconstructions were compared to the corresponding database profiles, and the difference profile in both cases were stored for statistical analysis. Six representative cases are presented in figure 9, which show examples of centrally peaked and hollow current profiles, positive and negative edge current (the latter relevant to rampdown scenarios) and small scale variations (the edge region of the $\left\langle j_{\phi}\right\rangle$ profile in case 2).

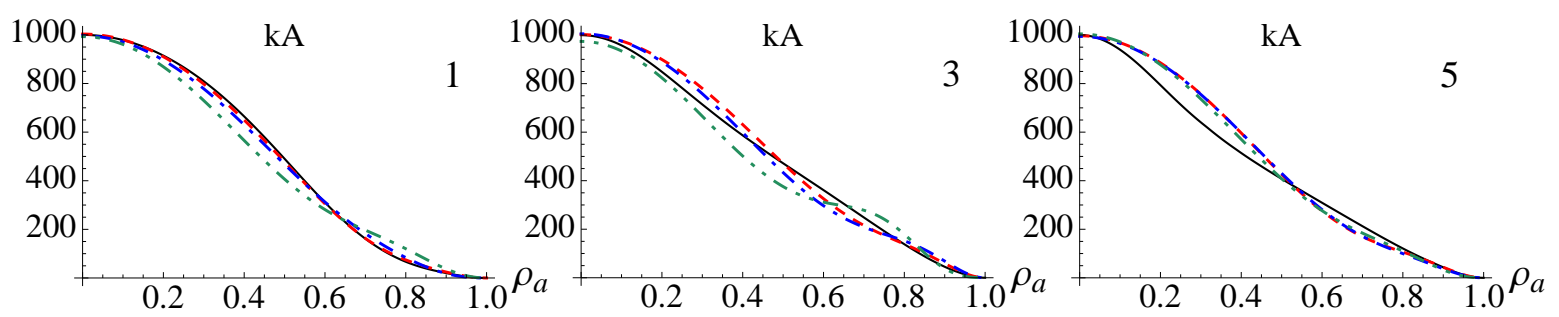

Figure 10. (colour online) Full exact and reconstructed $I_{\phi}$ profiles for cases 1,3 and 5 in figure 9.

The reconstruction error for $I_{\phi}$ profiles tends to zero as $\rho_{a} \rightarrow 0$ since $I_{\phi}(0) \equiv I_{p}$, the total plasma current, which is the most robustly determined parameter from magnetic data as can be seen in figure 10 which shows the full exact and reconstructed $I_{\phi}$ profiles for cases 1,3 and 5 in figure 9 . The rms reconstruction error for $I_{p}$ for all 600 cases in the database is $\delta_{r m s} I_{p}=3.4 \mathrm{kA}, 5.6 \mathrm{kA}, 8.2 \mathrm{kA}$ for the $\sigma=1 \mathrm{mT}, 2 \mathrm{mT}$ and $3 \mathrm{mT}$ noise levels, respectively.

In the six cases shown in figure 9, there are in general very large differences between the reconstructed and exact $\left\langle j_{\phi}\right\rangle$ profiles. However, in the edge region, and particularly for $\rho_{a}>0.95$ corresponding to $\rho_{\text {pol }} \gtrsim 0.985$, the reconstructed and exact profiles are more strongly correlated. The correlation in general weakens with increasing error level although the random nature of signal noise can lead to chance improvements as in case 4 where the reconstruction from signals perturbed with $\sigma=2 \mathrm{mT}$ noise almost overlays the exact profile for $\rho_{a}>0.94$. The reconstructed toroidal current profile for the lowest noise level is closely aligned to the exact profile for $\rho_{a}>0.95$ and, in particular, CLISTE in most cases successfully detects negative edge current when present.

The overall statistics are presented in figure 11 which includes results for noise levels of $\sigma=1 \mathrm{mT}, 2 \mathrm{mT}$ and $3 \mathrm{mT}$ for the edge region $\left\langle j_{\phi}\right\rangle$ and $I_{\phi}$ profiles. The rollover in the $\left\langle j_{\phi}\right\rangle\left(\rho_{a}\right)$ error for the $\sigma=2 \mathrm{mT}$ and $\sigma=3 \mathrm{mT}$ noise levels may be an artifact of the CLISTE spline regularization. The improvement in reconstruction quality with decreasing signal noise magnitude is evidence of the predictive power of magnetics, 

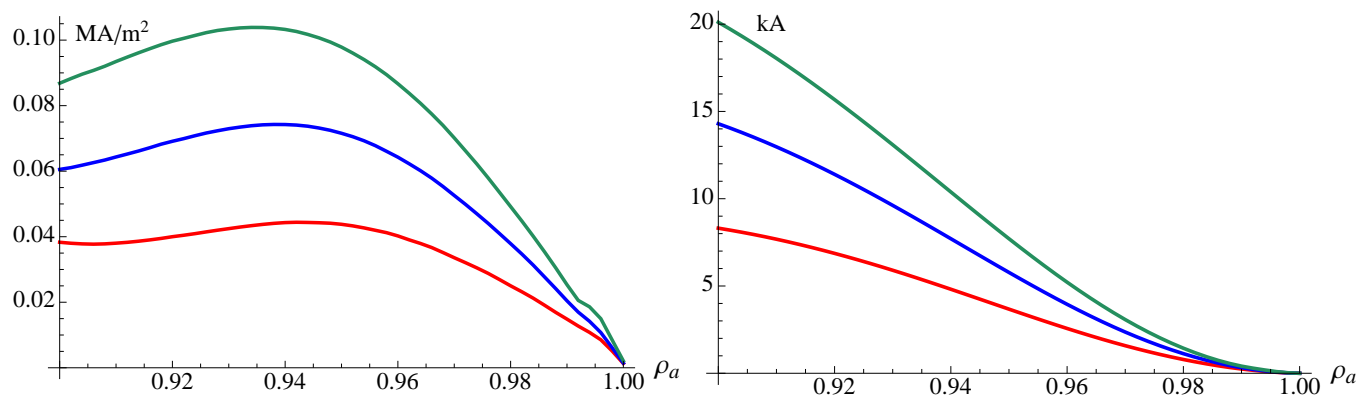

Figure 11. (colour online) Edge region root mean square reconstruction errors versus area radial parameter $\rho_{a}$ for flux surface averaged current density $\left\langle j_{\phi}\right\rangle$ (left-hand plot) and toroidal current $I_{\phi}$ profiles (right-hand plot). Results for $1 \sigma$ noise levels of $1 \mathrm{mT}$ (red traces), $2 \mathrm{mT}$ (blue), and $3 \mathrm{mT}$ (green) are included.

and supports the earlier results that equilibrium magnetic data contains information on the edge current profile. For ASDEX Upgrade 1MA discharges, well-fitted CLISTE reconstructions in flat-top have rms magnetics fit errors which typically lie in the range $1-1.5 \mathrm{mT}$, so that scaling up the $\sigma=1 \mathrm{mT}$ results presented here by a factor of 1.25 , which indicates a $1 \sigma$ uncertainty of $2.5 \mathrm{kA}$ at $\rho_{a}=0.965$ or $\left\langle\rho_{\text {pol }}\right\rangle=0.99$, provide a reasonable estimate of the underlying uncertainty in CLISTE reconstructions. If, as is the case on ASDEX Upgrade, the total toroidal current flowing in the SOL can be accurately inferred from divertor tile poloidal current data (as indicated by the tight constraints on the $j_{\phi}$ profile in the SOL region of figure 5), then the accuracy levels for $I_{\phi}$ in the edge region presented here and based on an equilibrium database without SOL currents should also hold for the more realistic case with SOL currents.

A selection of good quality CLISTE reconstructions spanning 8 years of operation was carried out, amounting to 4750 time points distributed over 104 ASDEX Upgrade discharges that satisfied $0.6 \leq I_{p} \leq 1.2 \mathrm{MA}$ and with an rms magnetics fit error, normalized for $I_{p}=1 \mathrm{MA}$, satisfying $\delta_{r m s}<1.5 \mathrm{mT}$. The following table shows a number of statistics for the toroidal current $I_{\phi}$ at the $\rho_{\text {pol }}=0.99$ flux surface, as well as the portion of $I_{\phi}\left(\rho_{\text {pol }}=0.99\right)$ flowing inside the separatrix. Statistics for the fitted toroidal SOL current are also presented. All three currents are normalized for $I_{p}=1 \mathrm{MA}$, and all values are rounded to the nearest $\mathrm{kA}$ per MA plasma current.

\begin{tabular}{|c|c|c|c|c|}
\hline & mean & std. deviation & minimum & maximum \\
\hline$I_{\phi, \text { tot }}\left(\rho_{\text {pol }}=0.99\right)$ & 40 & 14 & -7 & 90 \\
\hline$I_{\phi, \text { in }}\left(\rho_{\text {pol }}=0.99\right)$ & 18 & 7 & -12 & 46 \\
\hline$I_{S O L}$ & 23 & 12 & -19 & 71 \\
\hline
\end{tabular}

The estimated underlying $1 \sigma$ error of $2.5 \mathrm{kA}$ in the reconstructed value of $I_{\phi}\left(\rho_{\text {pol }}=0.99\right)$ thus represents a $6 \%$ uncertainty in the mean value obtained from this survey, or a $14 \%$ uncertainty in the mean value of $18 \mathrm{kA}$ flowing inside the separatrix. 


\section{Discussion and Conclusions}

In contrast to what has previously been widely accepted, the results presented here demonstrate both analytically and experimentally, and provide supportive statistical results from a randomly generated equilibrium database, that standard equilibrium magnetic measurements in a tokamak contain valuable information on the current density profile close to the boundary of an X-point plasma. This is at variance with the pessimistic conclusions of [11] which, however, have been shown here to have been unjustified since the main derivation in [11] does not take the force balance constraint into consideration.

The analytical examples combined with the qualitative consistency between figure 6(a) and 6(b) and the quantitative agreement between the $I_{\text {edge }}$ time traces in figures 8,9 and 11 allow the following conclusions to be drawn: Equilibrium magnetic measurements yield information on the $j_{\phi}$ profile near the boundary of an X-point plasma. Full details of the edge profile shape cannot be identified from magnetics alone, but the current flowing outside a reference flux surface near the separatrix, in the vicinity of $v=0.9$, or equivalently $\rho_{a}=0.965$ and $\rho_{\text {pol }}=0.99$, is determined to within a few $\mathrm{kA}$ for a $1 \mathrm{MA}$ discharge. The estimated $2.5 \mathrm{kA} 1 \sigma$ uncertainty in the total toroidal current at the $\rho_{\text {pol }}=0.99$ surface equates to a $6 \%$ error in the mean value from a large survey of CLISTE reconstructions. The fact that this region coincides with that of high pressure gradients and therefore high current densities associated with the edge transport barrier characteristic of the H-mode offers vital additional diagnostic information in the challenge to determine the experimental stability limits affecting ELM dynamics and to investigate wider issues of pedestal physics.

\section{Acknowledgments}

Fruitful discussions with Karl Lackner are cordially acknowledged. This work was supported by a research agreement between the Max Planck Institut für Plasmaphysik, Garching and the Department of Physics, University College Cork, and by EURATOM. The paper has benefitted significantly from constructive criticism by the anonymous referees.

\section{References}

[1] J L Luxon, B. B. Brown, Nucl. Fusion 22813 (1982).

[2] L L Lao, H. St.John et al., Nucl. Fusion 251611 (1985).

[3] L L Lao, J. R. Ferron et al., Nucl. Fusion 301035 (1990).

[4] J Svennson, A Dinklage, J Geiger et al., Rev. Sci. Instrum. 754219 (2004).

[5] M J Hole, G. von Nessi, D Pretty et al., Rev. Sci. Instrum. 81 10E127 (2010).

[6] D Mazon, A Murari, C Boulbe et al., Fusion Sci. Tech. 58742 (2010).

[7] K Lackner, Comput. Phys. Commun. 1233 (1976).

[8] Wolfram Research, Inc., Mathematica, Version 7.0, Champaign IL, (2008).

[9] B J Braams, Plas. Phys. Control. Fusion 33715 (1991). 
[10] L L Lao et al., Fusion Sci. Tech. 48968 (2005).

[11] V D Pustovitov, Nucl. Fusion 41721 (2001).

[12] P J Mc Carthy et al., IPP Report 5/85, Max Plank Institut für Plasmaphysik, 1999.

[13] P J Mc Carthy, Phys. Plas. 63554 (1999).

[14] P J Mc Carthy, Ph.D. thesis, University College Cork 164 (1992).

[15] W Schneider, P J Mc Carthy, K Lackner et al., Fusion Eng. Des. 48127 (2000).

[16] V A Morozov, Sov. Math.-Dokl. 7414 (1966).

[17] P J Mc Carthy, Inverse Problems 19643 (2003).

[18] P J Mc Carthy, F C Morabito, Int. J. App,. Electrom. Mechanics 8343 (1997).

[19] P J Mc Carthy, C B Forest, M Foley et al., 21st IAEA Fusion Energy Conference, Chengdu, China 16th-21st October 2006, TH/P3-7.

[20] A K Cline, Communications of the ACM 17218 (1974).

[21] A Kallenbach, A. Carlson, G. Pautasso et al., J. Nucl. Mater. 290639 (2001).

[22] M Reich, E. Wolfrum et al., Plas, Phys. Control. Fusion 46797 (2004).

[23] E Wolfrum, J. Schweinzer et al., Rev. Sc. Inst. 77033507 (2006).

[24] N K Hicks, W Suttrop et al., Fusion Sci. Tech. 571 (2010).

[25] B Kurzan, H Murmann, H Salzmann, Rev. Sc. Inst. 721111 (2001). 